\title{
Estimating the flood frequency distribution at seasonal and annual time scales
}

\author{
E. Baratti ${ }^{1}$, A. Montanari ${ }^{1}$, A. Castellarin ${ }^{1}$, J. L. Salinas ${ }^{2}$, A. Viglione $^{2}$, and A. Bezzi ${ }^{3}$ \\ ${ }^{1}$ School of Civil Engineering, Dept. DICAM, University of Bologna, Bologna, Italy \\ ${ }^{2}$ Institute of Hydraulic Engineering and Water Resources Management Vienna University of Technology, Vienna, Austria \\ ${ }^{3}$ Studio Ing. G. Pietrangeli S.r.l., Via Cicerone 28, Rome, Italy
}

Correspondence to: E. Baratti (emanuele.baratti@unibo.it)

Received: 31 May 2012 - Published in Hydrol. Earth Syst. Sci. Discuss.: 27 June 2012

Revised: 22 November 2012 - Accepted: 26 November 2012 - Published: 13 December 2012

\begin{abstract}
We propose an original approach to infer the flood frequency distribution at seasonal and annual time scale. Our purpose is to estimate the peak flow that is expected for an assigned return period $T$, independently of the season in which it occurs (i.e. annual flood frequency regime), as well as in different selected sub-yearly periods (i.e. seasonal flood frequency regime). While a huge literature exists on annual flood frequency analysis, few studies have focused on the estimation of seasonal flood frequencies despite the relevance of the issue, for instance when scheduling along the months of the year the construction phases of river engineering works directly interacting with the active river bed, like for instance dams. An approximate method for joint frequency analysis is presented here that guarantees consistency between fitted annual and seasonal distributions, i.e. the annual cumulative distribution is the product of the seasonal cumulative distribution functions, under the assumption of independence among floods in different seasons. In our method the parameters of the seasonal frequency distributions are fitted by maximising an objective function that accounts for the likelihoods of both seasonal and annual peaks. In contrast to previous studies, our procedure is conceived to allow the users to introduce subjective weights to the components of the objective function in order to emphasize the fitting of specific seasons or of the annual peak flow distribution. An application to the time series of the Blue Nile daily flows at the Sudan-Ethiopia border is presented.
\end{abstract}

\section{Introduction}

Flood frequency analysis is often used by practitioners to support the design of river engineering works, flood mitigation procedures and civil protection strategies. It is generally carried out by fitting peak flow observations to a suitable probability distribution. Two approaches are mainly applied. Using an annual maximum series (AM), one considers the largest event in each year. Conversely, using a partial duration series, or peak-over-threshold method (POT), the analysis considers all peaks above a given threshold level (e.g. Madsen et al., 1997a,b).

In many practical cases one may be interested in inferring the flood frequency distribution for given intra-annual periods. For instance, the design of a small coffer dam that will be in place for only a couple of months may be over-designed if the design flood is based on the AM analysis, especially if the period in which the coffer dam is in place is during a drier season of the year (e.g. McCuen and Beighley, 2003). When considering utilisation of floodwater for water supply it is very valuable to use seasonal flood information so as to operate the reservoir more effectively during flood seasons without increasing the flood risk (e.g. Fang et al., 2007; Chen et al., 2010).

There are several problems encountered when fitting seasonal and annual frequency curves independently and a key issue is to ensure the compatibility between intra-annual and annual flood probability distributions. One example is the problem of crossing over: in the probability plot, the annual distribution must always lie on or above the highest seasonal distribution (Durrans et al., 2003), i.e. the probability of 
one peak value of being exceeded in the entire year must be higher than the probability of the same value being exceeded in one season.

The issue of seasonal flood frequency analysis was considered by Creager et al. (1951). However, literature dedicated little attention to this problem in comparison with the estimation of annual extremes. In fact, several contributions dealt with intra-annual flood assessment but in many cases the purpose was to derive seasonal information for use in the estimation of the annual peak flow. For instance, Stedinger et al. (1992) discussed the advantages and drawbacks related to using seasonal flow data to estimate the annual peak flow distribution but did not explicitly focus on flood estimation in sub-yearly periods. Similarly, Kochanek et al. (2012) and Strupczewski et al. (2012) focused on the upper quantiles of the annual peak flows by fitting data collected in two seasons. Other analogous contributions were provided by Buishand and Demarè (1990), who refer to rainfall depths, and Singh et al. (2005).

Among the contributions that are explicitly dedicated to inferring flood occurrence in different seasons, it is worth mentioning McCuen and Beighley (2003), who focused on filling the gaps of seasonal data records, and in particular Durrans et al. (2003), who first considered alternative approaches to jointly estimate seasonal and annual flood frequency distributions. However, their methods are based on adapting the skewness coefficient of seasonal distributions to ensure a satisfactory fit of the annual peak flows, thereby putting more emphasis on the annual distribution. Allamano et al. (2011) analyse the magnitude of under- (or over-) estimation of design events in the presence of seasonality by using the POT or AM approach. Bowers et al. (2012) presents a statistical procedure to partition river flow data into three seasons and focuses on two particular distributions to describe the constructed seasonal river flows: power law and lognormal. Fang et al. (2007) proposed an approach based on the peaks-over-threshold sampling method and a nonidentical Poisson distribution to model the flood occurrence within each season. Another relevant contribution was recently given by Chen et al. (2010) who proposed the use of a copula function to jointly model the distributions of flood magnitude and date of occurrence.

We propose a practical and useful alternative approach for jointly estimating seasonal and annual flood frequency distributions, which has the relevant feature that, under the assumption of mutual independence of seasonal peaks, the number of seasons and their distribution along the year can be defined with great flexibility. In detail, we analyse yearly maxima collected at seasonal and annual time scales and develop an objective function for parameter estimation, consisting of the weighted sum of seasonal and annual loglikelihoods for the peaks of being observed. Parameters of the seasonal distributions are optimised while, under the assumption of independence of the flood generating process among seasons, the annual distribution is computed as the product of the seasonal ones. Likelihood weights can be used to put more emphasis on one or more distributions, whether sub-yearly or annual. It is worth noting that the optimisation procedure is similar to a maximum likelihood estimation, but our objective function is not a likelihood function since it combines seasonal and annual likelihoods and it allows the user to assign weights to them. The method represents an approximate solution to the problem of the seasonal and annual flood frequency analysis, providing results that are wholly comparable to those that would be obtained through the classic method of annual, or seasonal, peak discharge and overcoming consistency problems such as the crossing over problem.

With respect to the approach proposed by Chen et al. (2010) our method ensures more flexibility in the choice of the seasons which can eventually be very different in terms of their impact on the annual flood distribution. For illustration purposes, the proposed approach is applied to infer seasonal and annual flood frequency distributions for the Nile River at the Sudan-Ethiopia border.

\section{Parameterisation of seasonal and annual flood frequency distributions}

Let us define a season as a contiguous period of the year with its own river flow regime and seasonal flood frequency distribution. Assuming that the year is divided into $N$ seasons in which flood distributions are independent of each other, the cumulative probability distribution function (CDF) of the annual maximum flood $F_{Q_{Y}}$ is given by the product (e.g. Waylen and Woo, 1982; Durrans et al., 2003)

$$
F_{Q_{Y}}\left(q \mid\left(\boldsymbol{\theta}_{1}, \ldots, \boldsymbol{\theta}_{N}\right)\right)=\prod_{i=1}^{N} F_{Q_{i}}\left(q \mid \boldsymbol{\theta}_{i}\right), \quad i=1, \ldots, N
$$

where $F_{Q_{i}}$ and $\boldsymbol{\theta}_{i}$ are the $\mathrm{CDF}$ and the vector of the parameters for season $i$, respectively. This relationship clearly shows that seasonal and annual probability distributions are strictly related, meaning that the estimation of their parameters should be conditioned by Eq. (1). The literature has proposed several methods to impose the aforementioned condition. For instance, Durrans et al. (2003) conditioned the skewness coefficients of seasonal distributions to fit the annual flood frequency behaviours.

Under the assumption of independence among seasonal peak flows, we propose an estimation technique for seasonal and annual flood frequency distributions which enables the user to (a) select the seasons independently of their significance in the formation of the overall flood regime, (b) assign different weights to the fitting of seasonal and annual distributions and (c) overcome the problem of the crossing over among seasonal and annual distributions.

The method makes use of the annual maxima sampling method to select relevant floods at seasonal and annual time scale and is articulated in the following steps. 
1. From the observed data series, select the sample of the annual maximum peak flows (AM) as well as the samples of annual maxima for each season $\left(\mathrm{SM}_{i}\right)$.

2. For the above seasonal samples, identify a suitable probability distribution and estimate its parameters, for instance by using the method of maximum likelihood (i.e. initial parameter set).

3. Compute the objective function $\xi\left(\boldsymbol{\theta}_{1}, \ldots, \boldsymbol{\theta}_{N}\right)$ for the joint-estimation of seasonal and annual distributions parameters through the relationship

$$
\begin{array}{r}
\xi\left(\boldsymbol{\theta}_{1}, \ldots, \boldsymbol{\theta}_{N}\right)=\sum_{i=1}^{N}\left\{w_{i} \sum_{j=1}^{M_{S_{i}}} \ln \left[f_{Q_{i}}\left(q_{S_{i, j}} \mid \boldsymbol{\theta}_{i}\right)\right]\right\} \\
+w_{Y} \sum_{k=1}^{M_{Y}} \ln \left[f_{Q_{Y}}\left(q_{Y, k} \mid\left(\boldsymbol{\theta}_{1}, \ldots, \boldsymbol{\theta}_{N}\right)\right)\right]
\end{array}
$$

where $M_{S_{i}}$ and $M_{Y}$ are the samples sizes of $\mathrm{SM}_{i}$ and $\mathrm{AM}$, respectively, which may be different (e.g. the case of seasons affected by missing data); $q_{S_{i, j}}$ and $q_{Y, k}$ are the observations in $\mathrm{SM}_{i}$ and $\mathrm{AM}$, respectively; $f_{Q_{i}}\left(q_{S_{i, j}} \mid \boldsymbol{\theta}_{i}\right)$ and $f_{Q_{Y}}\left(q_{Y, k} \mid \boldsymbol{\theta}_{1}, \ldots \boldsymbol{\theta}_{N}\right)$ are the seasonal and annual probability density functions; $w_{i}$ and $w_{Y}$, with $w_{Y}+\sum_{i=1}^{N} w_{i}=1$ are weights, for the seasonal and annual distributions, respectively. Note that Eq. (2) is based on the log-likelihood function of the seasonal and annual distributions given the observed intra-annual and annual peak discharges. In view of Eq. (1), the annual log-likelihood, presents in the second term at the right hand side of Eq. (2), can be computed by

$$
\begin{aligned}
& \ln \left[f_{Q_{Y}}\left(q_{Y, k} \mid\left(\boldsymbol{\theta}_{1}, \ldots, \boldsymbol{\theta}_{N}\right)\right)\right] \\
& =\ln \left[\sum_{i=1}^{N} f_{Q_{i}}\left(q_{Y, k} \mid \boldsymbol{\theta}_{i}\right)\left(\prod_{\substack{j=1 \\
j \neq i}}^{N} F_{Q_{J}}\left(q_{Y, k} \mid \boldsymbol{\theta}_{j}\right)\right)\right] .
\end{aligned}
$$

Since Eq. (2) depends only on seasonal parameters $\boldsymbol{\theta}_{1}, \ldots, \boldsymbol{\theta}_{N}$.

4. Through an optimisation algorithm, the objective function given by Eq. (2) is maximised in order to obtain the best parameter set (i.e. optimal parameter set).

Weights in Eq. (2) are introduced to control the relevance of the fit of each single frequency distribution in the overall procedure. With a proper choice of weights, the proposed procedure can converge to the traditional procedure that is based on the analysis of annual maxima only. One application of the proposed approach is presented in the following section.

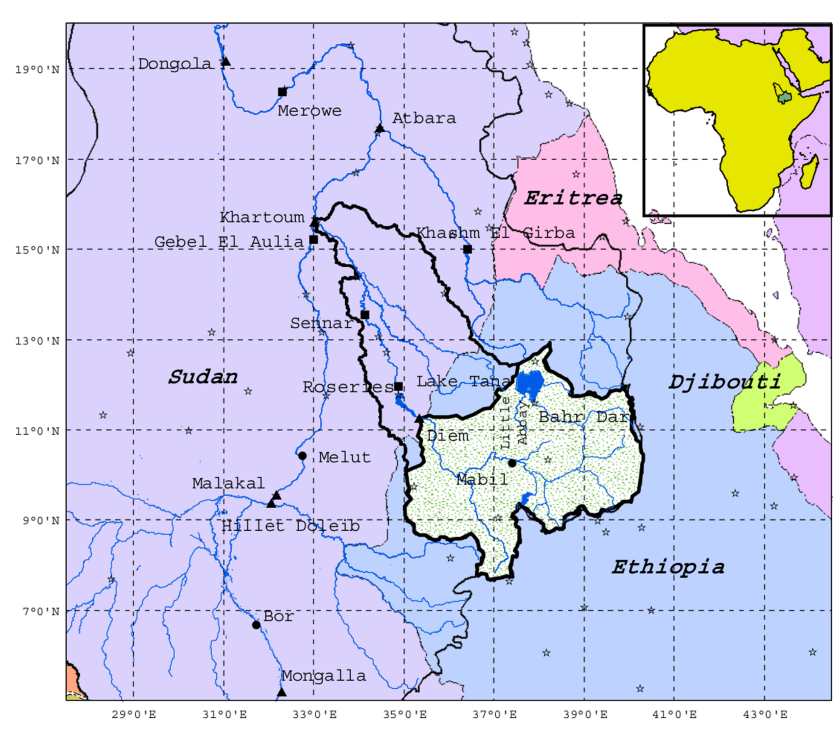

Fig. 1. Schematic representation of the upper Blue Nile Basin (hatched) within Blue Nile River basin (outlined). Source: Fig. 1 in Elshamy et al. (2009).

\section{Application to the Blue Nile River at Sudan-Ethiopia Border}

The proposed method was used to estimate the flood frequency distribution, by referring to different yearly subperiods, for the Blue Nile River at the Sudan-Ethiopia Border. The Blue Nile originates from Lake Tana, in Ethiopia. Together with the White Nile, it is one of the major tributaries of the Nile River. The main stream length at Sudan Border is about $900 \mathrm{~km}$ and the contributing area is $175000 \mathrm{~km}^{2}$. The Blue Nile is vital to the livelihood of Egypt. In fact, about $59 \%$ of the water that reaches Egypt, originates from the Blue Nile. Figure 1 shows a schematic representation of the Blue Nile watershed.

Daily river flow observations that were collected by the Ethiopian Ministry of Irrigation and Water Resources (MoWR) between 1961 and 2005 (i.e. 45 yr) are available at Sudan Border. Nevertheless, the series is affected by several missing data, mainly from January to June/July, therefore the number of usable years is less than 45 . In particular, we retained in the annual maximum series of flood flows only the $25 \mathrm{yr}$ for which daily observations are available during the whole wet season (i.e. from June to September, see Rientjes et al., 2011). Concerning seasonal sub-samples, we included the seasonal maximum daily discharge in the seasonal database only when observations are available for at least $70 \%$ of the $i$-th season.

The maximum annual flood of the Blue Nile exhibits a very strong seasonality and is characterised by one peak season, as it is common in monsoon-dominated climates. Indications reported in the literature (e.g. Rientjes et al., 2011) distinguishes two main climatic seasons for the study 


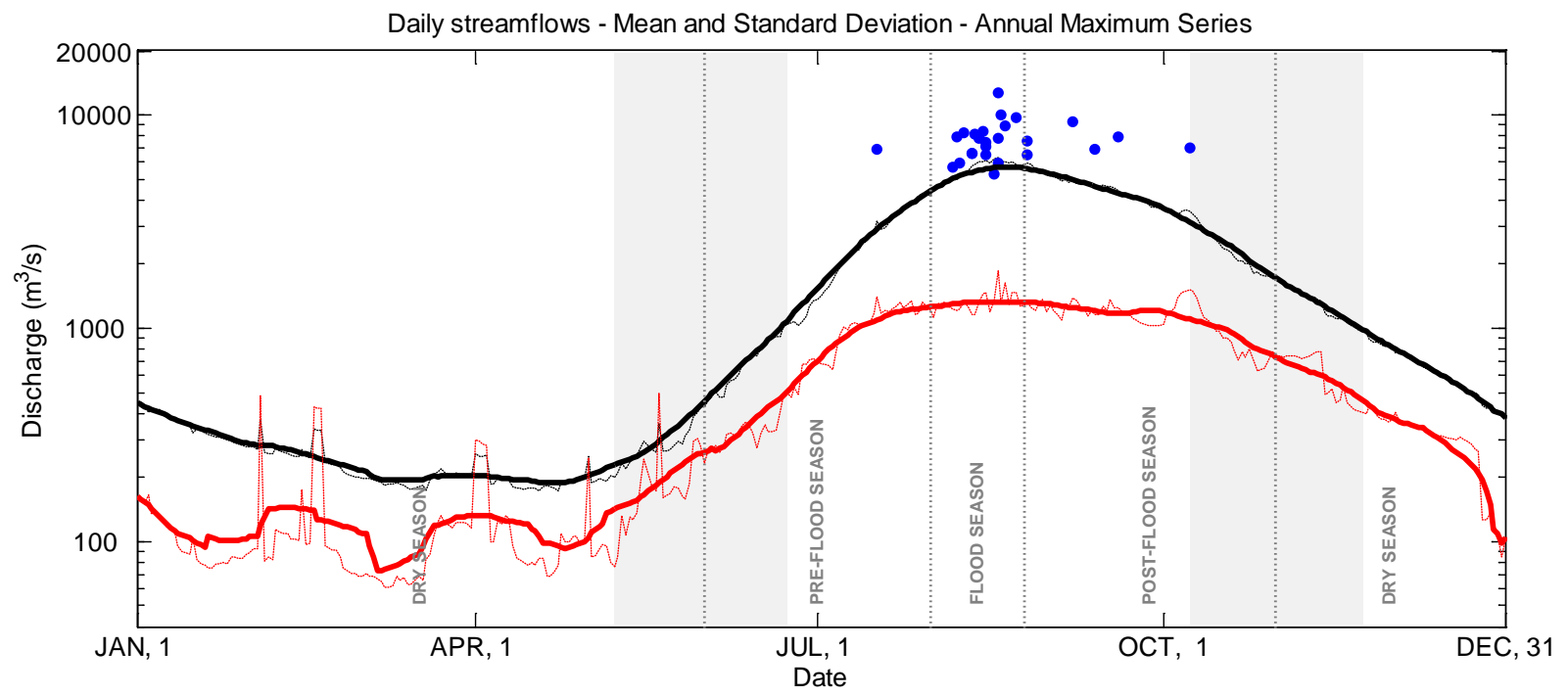

Fig. 2. Mean daily streamflow (black thin dotted line) and 30-day running mean (black thick line); standard deviation of daily streamflows (red thin dotted line) and a 30-day running mean (red thick line) as a function of the day of the year; annual maximum series are reported as blue dots; the four seasons considered in the study are also illustrated (grey shaded areas represent the time intervals considered in the sensitivity analysis, see Sect. 4.2).

area, namely: a wet (i.e. from June to September) and a dry (i.e. from October to May) season, while in the easternmost part of the study area a subdivision into three climatic seasons is suggested by some authors (e.g. Seleshi and Camberlin, 2006): Kiremt ("main rains", heavy rainy season, June-September), Belg ("small rains", light rainy season, February-May), and the dry season, Bega (OctoberJanuary). During the wet season the contribution of the Blue Nile is about two thirds of the total flow of the receiving Nile River.

\subsection{Season identification}

In order to identify the optimal number of seasons, climatic behaviours were considered along with the practical need to estimate peak flows for assigned periods for water resources management purposes. To obtain a first picture of climatic behaviours, Fig. 2 shows the progress of the mean daily streamflows along the year (black thin dotted line) together with a 30-day running mean (black thick line). The standard deviation of daily streamflows is also reported (red thin dotted line) together with a 30-day running mean (red thick line), while annual maximum peak flows are indicated as blue dots. It can be seen that seasonality is very pronounced with one flood season only.

Directional statistics were used to quantify seasonality of flood events (Mardia, 1972) on the basis of the timing of annual maximum flood flows. The utilisation of these seasonal indicators in hydrology is not new. For instance, the literature reports several effective applications in regional flood frequency analysis for explaining hydrologic similarity among catchments (e.g. Burn, 1997; Castellarin et al., 2001; Cunder- lik and Burn , 2002). After Bayliss and Jones (1993), the date of occurrence of the event $i$ can be written as a directional statistic by converting the Julian date, Jd, of occurrence into an angular measure given by

$\varphi_{i}=\mathrm{Jd}_{i}\left(\frac{2 \pi}{365}\right)$.

Therefore, each date of occurrence can be represented in polar coordinates as a vector with a unit magnitude and a direction given by Eq. (4). This allows the determination of the $x$ and $y$ coordinates of the mean of a sample of $Z$ dates of occurrence as

$x=\frac{1}{Z} \sum_{i=1}^{Z} \cos \left(\varphi_{i}\right) ; \quad y=\frac{1}{Z} \sum_{i=1}^{Z} \sin \left(\varphi_{i}\right)$.

Therefore, the direction $\bar{\varphi}$, along with the magnitude, $r$, of the vector representing this point in polar coordinates, can be obtained by

$\bar{\varphi}=\arctan \left(\frac{y}{x}\right)$,

$r=\sqrt{x^{2}+y^{2}}$.

Equation (6) represents a measure of the mean timing for the sample of $Z$ dates, such as the days of occurrence in an annual maximum series, and can be converted back to a mean date, MD, through

$\mathrm{MD}=\bar{\varphi}\left(\frac{365}{2 \varphi}\right)$.

Equation (7) gives a measure of the regularity of the phenomenon: values of $r$ close to one imply a strong seasonality, 
Table 1. Pearson's seasonal correlation coefficients for the Blue Nile River at Sudan Border. The second to last column reports the linear correlation coefficient between seasonal flood flows and the associated Gumbel reduced variate; the last column shows the PPCC test value for assessing the goodness of the fit of the Gumbel (i.e. EV1) distribution for a significance level of $5 \%$.

\begin{tabular}{|c|c|c|c|c|c|c|c|}
\hline \multirow[b]{2}{*}{ Season } & \multirow{2}{*}{$\begin{array}{l}\text { Record } \\
\text { length } \\
\text { (years) }\end{array}$} & \multicolumn{4}{|c|}{ Pearson's Corr. Coeff. } & \multicolumn{2}{|c|}{ PPCC } \\
\hline & & $\begin{array}{c}\text { Dry } \\
\text { Season }\end{array}$ & $\begin{array}{c}\text { Pre-flood } \\
\text { Season }\end{array}$ & $\begin{array}{l}\text { Flood } \\
\text { Season }\end{array}$ & $\begin{array}{c}\text { Post-flood } \\
\text { Season }\end{array}$ & $\begin{array}{l}\text { Corr. } \\
\text { coeff. }\end{array}$ & $\begin{array}{c}\text { Test value } \\
\alpha_{5 \%}\end{array}$ \\
\hline Dry & 18 & 1 & -0.018 & 0.000 & 0.355 & 0.972 & 0.933 \\
\hline Pre-flood & 21 & - & 1 & 0.008 & 0.158 & 0.977 & 0.940 \\
\hline Flood & 25 & - & - & 1 & 0.526 & 0.985 & 0.946 \\
\hline Post-flood & 24 & - & - & - & 1 & 0.982 & 0.944 \\
\hline AMS & 25 & & & & & 0.977 & 0.946 \\
\hline
\end{tabular}

or regularity, in the dates of occurrence of the events, values close to zero are symptomatic of a great dispersion throughout the year.

Through directional statistics the limits of the flood season, $\varphi_{1}$ and $\varphi_{2}$, are quantitatively identified through the relationship

$\varphi_{1,2}=\bar{\varphi} \pm \sigma$

where the negative and positive signs correspond to the beginning and the end of the season, respectively, and $\sigma$ is the standard deviation in radiants given by $\sigma=\sqrt{-2 \ln (r)}$ (Mardia, 1972). The computed mean angular measures are converted back to calendar dates by using Eq. (8). Directional statistics were applied to annual maximum series (AM) of daily streamflows of the Blue Nile at Sudan-Ethiopia Border. The results show that the annual maximum flood is extremely regular, with measure of regularity equal to $r=0.977$ (it is relevant to note that $r=1$ would correspond to observed annual maxima happening on the same day of the year). Most of the observed flood dates falls within the 3-week time period from 31 July to 25 August. We identify this period as the flood season. Furthermore, we identify three additional seasons to fully characterise the high streamflow regime (see Fig. 2), by also taking practical need of water resources management into account: a dry season from 1 November to 31 May; a pre-flood season from 1 June to 30 July, and a post-flood season from 26 August to 31 October. For each of the above periods, the seasonal maximum daily discharge was extracted. Table 1 presents the record length in years of each seasonal sample as well as the unique sample composed by the annual peak discharge. The matrix of the Pearson's correlation coefficients among the different samples containing the seasonal flood maxima is also shown in Table 1.

It can be seen that the null hypothesis of statistical mutual independence of seasonal peaks cannot be rejected at the $5 \%$ significance level, with the exception of the post-flood season that is positively correlated to the flood season.

It is relevant to point out that, in principle, the number of seasons and their calendar limits can be defined arbitrarily. However, for increasing number of seasons one experiences a
Table 2. Parameters of the seasonal distributions $\left[\mathrm{m}^{3} \mathrm{~s}^{-1}\right]$ : independent fitting (maximum likelihood estimation) and joint estimation by adopting uniform weights (proposed method).

\begin{tabular}{lccccr}
\hline \multirow{2}{*}{ Season } & \multicolumn{2}{c}{$\begin{array}{c}\text { Independent estimation } \\
\text { (Max. likelihood) }\end{array}$} & & \multicolumn{2}{c}{$\begin{array}{c}\text { Joint estimation } \\
\text { (Proposed method) }\end{array}$} \\
\cline { 2 - 3 } \cline { 6 - 7 } & Location & Scale & & Location & Scale \\
\hline Dry season & 1195 & 492 & & 1195 & 492 \\
Pre-flood season & 4446 & 1070 & & 4432 & 1056 \\
Flood season & 6694 & 1316 & & 6600 & 1267 \\
Post-flood season & 5668 & 1244 & & 5622 & 1208 \\
\hline
\end{tabular}

higher chance of detecting correlation among them. It is also significant to note that season identification based on climatic behaviours, rather than an arbitrary selection, leads to yearly subperiods that are well distinguished from a climatic point of view and therefore are more likely to be independent (e.g. Waylen and Woo, 1982; Durrans et al., 2003; Strupczewski et al., 2012; Kochanek et al., 2012).

\subsection{Estimation of seasonal and annual flood frequency distributions}

Table 1 reports the results of the Plotting Position Correlation Coefficient (PPCC) test (e.g. Vogel, 1986; Castellarin et al., 2004) that was carried out to test the suitability of the Gumbel distribution (also called EV1 distribution) to simulate the flood frequency behaviours in each season. For all seasons the linear correlation coefficient is reported between seasonal flood flows and their sample nonexceedance probability expressed through the Gumbel reduced variate (i.e. $y=-\ln (-\ln (F)$ ), where $F$ indicates the non-exceedance probability). The test value for a $5 \%$ significance level is also given. It can be seen that the Gumbel distribution can never be rejected at the $5 \%$ significance level for all the seasons. Therefore, initial values for the distribution parameters were estimated through the method of maximum likelihood (see Table 2). 


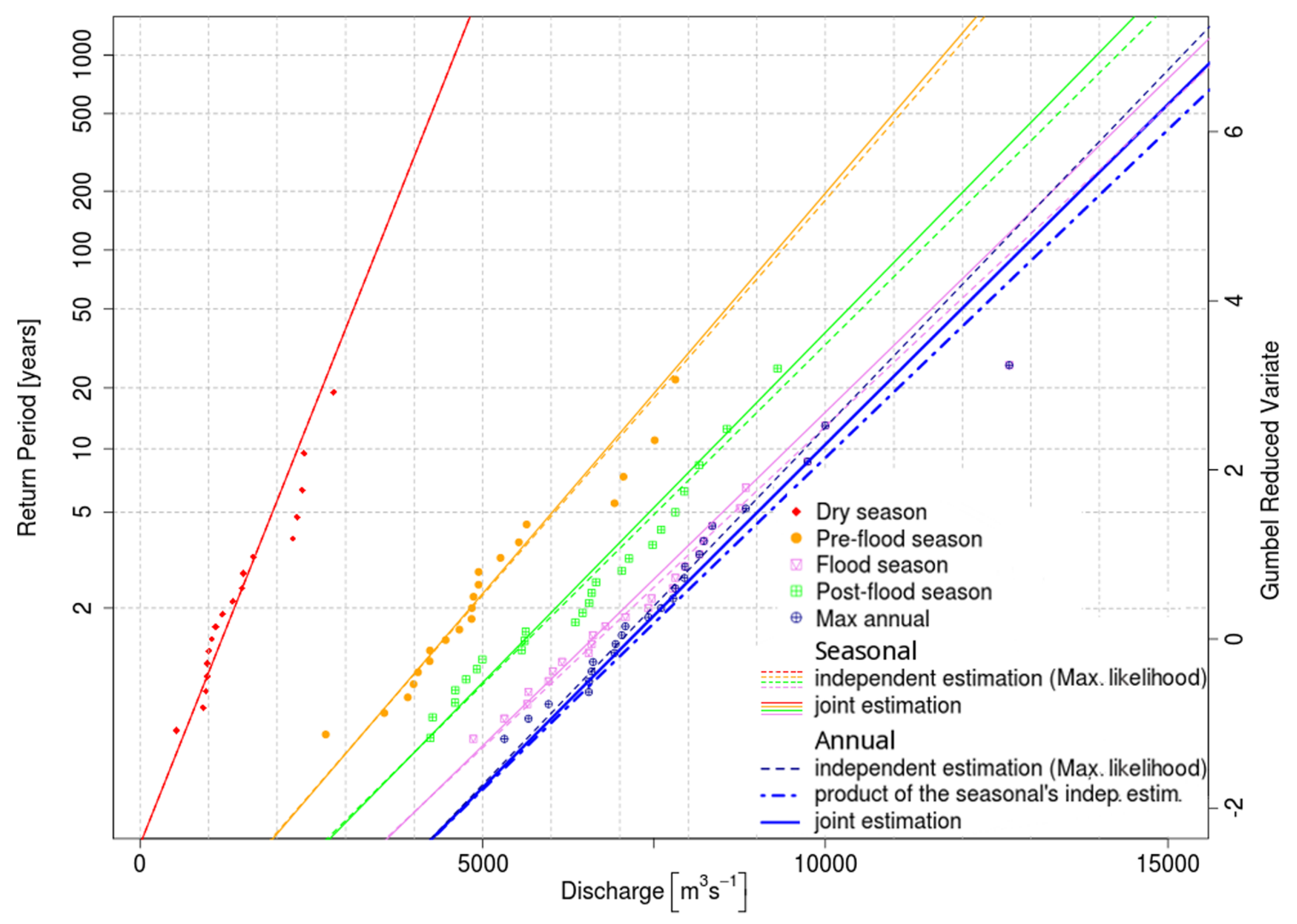

Fig. 3. Empirical CDFs (dots) and maximum likelihood estimates (dotted lines), blue refers to annual maxima; annual maximum CDF obtained as the product of the seasonal CDFs estimated through the method of maximum likelihood (blue dash-dotted lines); seasonal CDFs jointly estimated by maximizing Eq. (2) (thin lines) and annual flood CDF obtained as the product of the jointly estimated seasonal CDFs (thick blue line).

Through a genetic algorithm (Mebane and Sekhon, 2011), the seasonal distribution parameters were obtained by maximizing Eq. (2). Figure 3 shows in a Gumbel probability plot the initially fitted seasonal distributions (dotted lines) along with the sample frequency of the observed data (dots) and the final seasonal distributions resulting from the proposed approach (continuous lines) by adopting uniform weights. The annual CDF obtained from individual fitting of annual maxima and the ones obtained as the product of the initially fitted seasonal distributions are also shown (blue dotted and dashdotted lines, respectively). The differences are rather evident and result from the dependence between seasonal and annual peak-flow distributions. This dependence implies that the annual flood quantile estimated from seasonal maxima through the product of seasonal CDFs differs in general from the result of an at-site estimation focusing on the sequence of annual maximum discharges only. This is mainly due to the limited sample size of the considered sample, which results in discrepancies that increase with the return period. The annual distribution resulting from the joint estimation technique is also shown in Fig. 3 (blue continuous line).

Figure 3 highlights the problem of crossing over: for a return period approximately equal to $7-15 \mathrm{yr}$, the indepen- dently fitted flood season distribution (pink dotted line) cross the independently fitted annual distribution (blue dotted line). The seasonal distribution parameters, given by initial fitting and jointly fitting, are summarised in Table 2 .

Figure 3 shows a fair-to-good agreement between sample, individually fitted and joint estimated distributions, although some slight discrepancy is detected, as expected. In particular, the individually (independently) fitted seasonal distributions are underestimated while the contrary holds for the individually fitted annual distribution.

It can be also seen that the effect of dependence on the fitting of the post-flood and the flood season, namely, the difference between independent and joint estimates, is indeed negligible. As it was expected for the given season separation, it can be seen that the annual distribution is similar to that of the dominant flood season (Strupczewski et al., 2012; Kochanek et al., 2012). This can be explained by noticing that the majority of the annual maxima occurs during the flood season (i.e. from 31 July to 25 August), thus the samples of maximum peak flows collected for this season and at annual time scale are quite similar. 

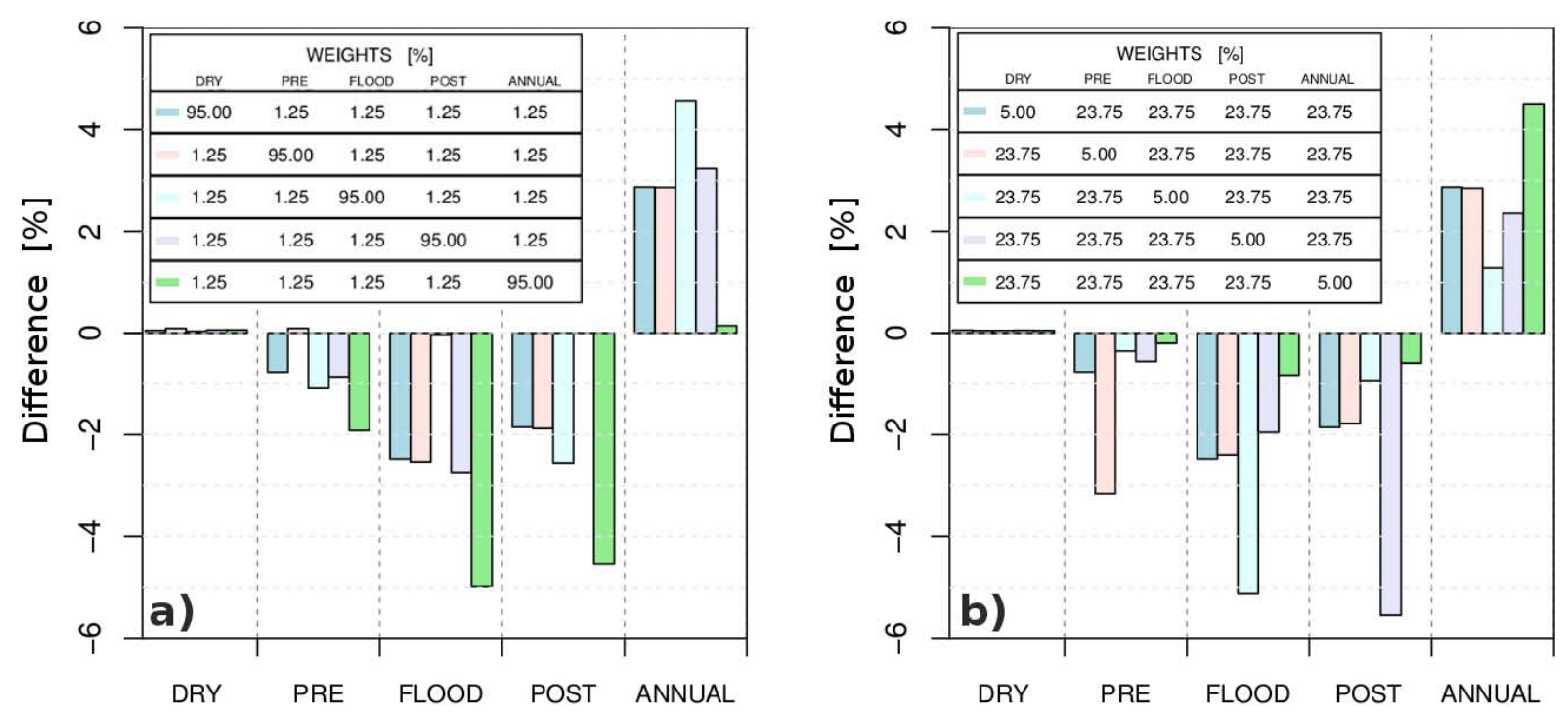

Fig. 4. Differences in percentage between the 100-yr peak flows estimated jointly (proposed technique) and the ones estimated by maximum likelihood independently on each season and on the maximum annual values. (a) Prevailing weight (95\%) to one given period and $1.25 \%$ to the other distributions; (b) small weight (5\%) to one given period and $23.75 \%$ to the other distributions.

\section{Sensitivity analysis}

This section illustrates some additional analyses we performed aiming at assessing the sensitivity of the proposed approach to three different aspects, namely (1) the seasonal weighting scheme used in the optimisation procedure; (2) the duration of seasons; (3) the number of seasons and hypothesis of independence between these.

\subsection{Sensitivity to the weighting scheme}

In Fig. 4 the sensitivity of the method to the choice of weights is characterised by showing the differences in percentage between the 100-yr peak flows estimated jointly with the proposed technique and the ones estimated by maximum likelihood independently on each season and on the maximum annual values.

The effect of attributing a prevailing weight $(95 \%)$ to one given season or to the annual distribution is shown in Fig. 4a. The estimated 100-yr quantile for the season (or annual distribution) with the high weight is very close to the corresponding independent estimate. This result is expected because the objective function is very close to the individual $\log$-likelihood function for the season (or annual distribution). On the other hand, Fig. 4b shows the effect of attributing a small weight (5\%) to one distribution only. For instance, if the small weight is assigned to the annual distribution (light-green weighs combination), the seasonal estimates lie close to the individual maximum likelihood estimates, since the objective function is almost coincident with the sum of the log-likelihoods of observing the seasonal peaks independently.
In summary, Fig. 4 demonstrates that the annual distribution, as well as the flood and post-flood seasons, are sensitive to the value of the weights, while the pre-flood and dry seasons display little or no sensitivity. This is because our method accounts for the dependence between seasonal and annual maxima (and not among the seasons themselves) and the peaks in the pre-flood and dry season almost never are maximum annual peaks.

\subsection{Sensitivity to the duration of seasons}

As shown in the previous section, we used an objective technique (i.e. directional statistics) to identify the flood season, while we referred to indications reported in the scientific literature for identifying the limits of the remaining three seasons. One may argue that these indications are not objective for the considered case study.

In order to quantify the sensitivity of the proposed approach to the duration of each season we performed a numerical experiment in which, we randomly changed the calendar limits of the seasons 1000 times. In particular, while we kept the limits of the flood season constant, we randomly selected the beginning of the pre-flood season and the end of the postflood season in each simulation. Julian dates were generated from a uniform distribution in an interval of one month and a half (i.e. 46 days) around the limit dates depicted in Fig. 2, that is \pm 23 days around 1 June, or Julian day 152, for the beginning of the pre-flood season, and around 31 October, or Julian day 304, for the end of the post-flood season (see grey shaded areas in Fig. 2).

Using these randomly identified seasons we extracted the seasonal peak flow samples and applied the proposed 
procedure (weights were all set equal to 0.2 as in Sect. 3.2) to jointly estimate the 100-yr flood quantile for the flood season (i.e. the season with fixed dates), the other seasons (variable starting and ending dates) and at annual time scale. We then computed the differences between the 100-yr flood quantiles jointly estimated for the randomly selected seasons and we compared them with the corresponding quantiles resulting from the application of the procedure to the four seasons defined in Sect. 3.1. As expected, the differences between estimated flood quantiles are always within the interval $\pm 1.3 \%$, except for the dry season. This can be explained with the strong seasonality of streamflows, since a wide variation of the dry-season calendar limits results in a remarkable change of the corresponding seasonal sample of peak flows. Nevertheless, when the proposed approach is applied to jointly estimate the seasonal flood frequency distributions, the remarkable changes in the dry season do not affect the results in terms of estimated 100-yr flood quantiles for the flood season (whose limits were kept constant in the 1000 random sampling) and the annual 100-yr flood quantile, which is a positive aspect of the proposed approach.

\subsection{Sensitivity to the number of seasons and hypothesis of independence among these}

As already discussed, the main assumption of the proposed approach is the hypothesis of independence among seasons. The degree of dependence is inevitably associated with the subdivision into season (i.e. number and duration of seasons). However, in some applications a practitioner may have to refer to a particular subdivision that is important for his/her engineering problem or decision-making process and cannot select seasons that are statistically independent. Moreover, the streamflow regime may not always be so clearly seasonal as in the Blue Nile case, where a well defined subdivision into wet and dry seasons exists.

In order to quantify the sensitivity of the proposed approach to the number of seasons and the associated hypothesis of independence, we considered two different numbers of season (i.e. two and four intra-annual periods) and, similarly to the previous section, we performed 1000 random subdivisions of the year into seasons. In particular, in the case of two seasons we subdivided the year into a wet (i.e. merger of pre-flood, flood and post-flood season, see Fig. 2) and a dry period, then we varied the limits between the two seasons exactly in the same way described in the previous subsection and in Fig. 2. In the case of four seasons, unlike the numerical experiment described in the previous section, we varied also the limits of the flood season, which we randomly selected in an interval of two weeks around the limits obtained through the directional statistic techniques (i.e. \pm 7 days around the 31 July and 25 August) to allow for the identification of combination of seasons with different degrees of statistical dependence. We then checked the significance of the hypothesis of statistical independence of seasons for each subdivision and we recorded the pairs of seasons showing statistically significant correlation coefficients. We estimated and compared the 100-yr flood quantiles (all weights are equal to 0.2 as in Sect. 3.2). In particular, in the case of four seasons we focused on the degree of statistical dependence between the flood and post-flood seasons, which was the only pair of seasons that showed a significant Pearson's correlation coefficient for the subdivision selected in the study (see Sect. 3.1).

Concerning the case of two seasons, none of the 1000 subdivisions resulted in a statistically significant dependence between the wet and dry periods, while, since all the annual maximum flood dates fell within the wet season, the sequences of seasonal maxima for the wet season and the annual maxima were the same. Therefore, (1) the main assumption of the proposed approach holds for all 1000 randomly selected seasonal samples and, as a consequence, (2) the differences between the 100-yr quantiles estimated with the proposed approach or independently for each time-period with the maximum likelihood estimator are negligible at seasonal and annual time scale.

Concerning the case of four seasons, the hypothesis of independence of seasonal peaks could not be rejected at the $5 \%$ significance level for 16 random subdivisions only; in 564 cases out of 1000 the Pearson's correlation coefficient was found to be statistically significant only between the flood and post-flood seasons; in the 420 remaining cases the post-flood season showed a statistically significant correlation with the flood and dry seasons. The results of the numerical experiment are illustrated in Fig. 5. The two panels of the figure report the results for the 16 random subdivisions showing no significant dependence between the seasons (panel a), and for the 564 cases in which the hypothesis of independence between the flood and post-flood seasons should be rejected at the $5 \%$ significance level (panel b).

Boxplots of Fig. 5 show the relative differences between the 100-yr peak flows estimated with the proposed method relative to the ones estimated independently at seasonal and annual time scale through the maximum likelihood method. Concerning the annual flood quantile the figure presents two boxplots, one refers to the estimation of the annual distribution as the product of the independently estimated seasonal CDFs (light blue boxplot), while the other refers to the joint estimation with the proposed method (blue boxplot). Figure 5 clearly shows that the bias increases when the hypothesis of independence does not hold in the strict sense (comparison between panels a and b). Also, Fig. 5 clearly points out that estimating the CDF of annual maxima as the product of the jointly estimated seasonal CDFs (blue boxplot) produces a better representation of the annual flood frequency regime relative to estimating the $\mathrm{CDF}$ of annual maxima as the product of the seasonal CDFs estimated separately from each other through the maximum likelihood methods (light blue boxplot). This consideration holds when the hypothesis of independence among seasons does not hold in the strict sense (panel b), but also, and this is perhaps more interesting, 

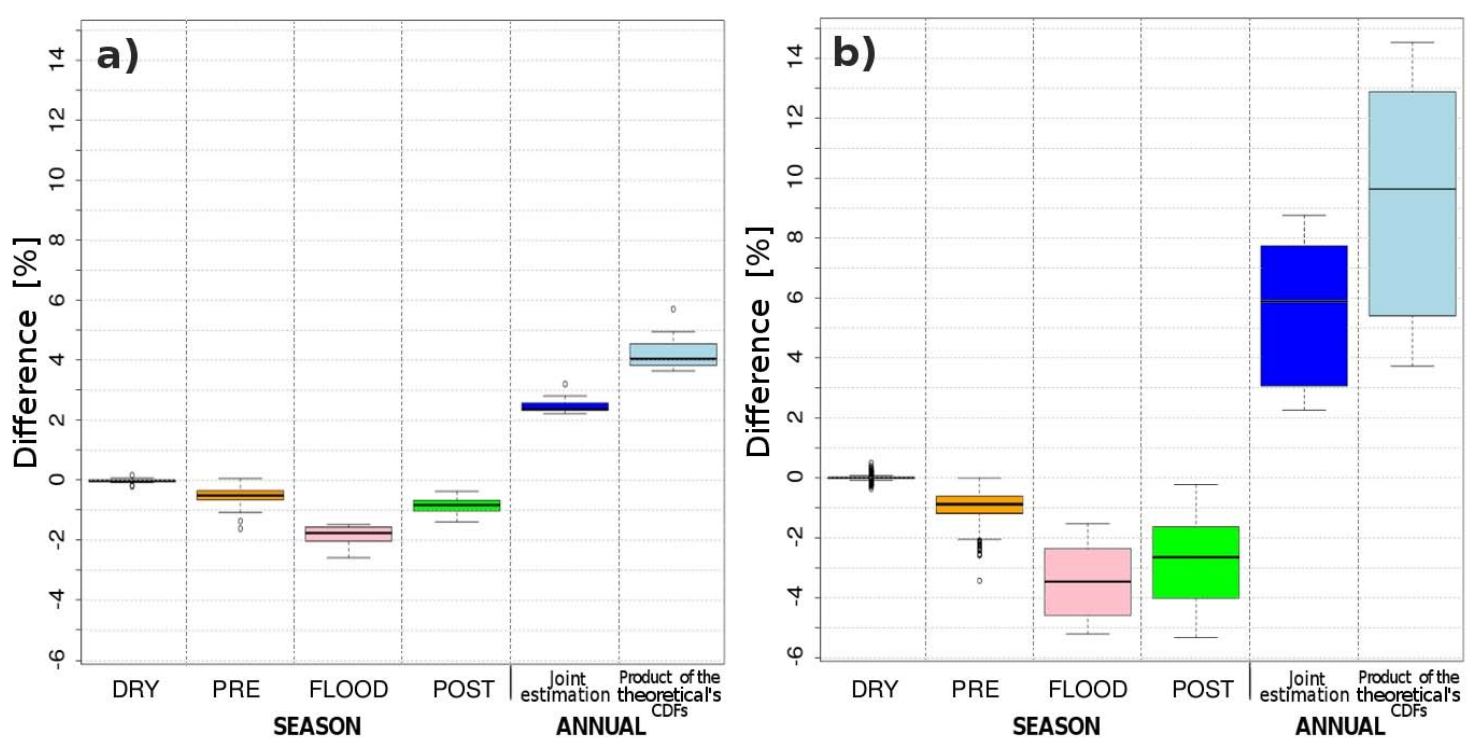

Fig. 5. Sensitivity of the proposed approach to the duration of and dependence among seasons (independent seasons, panel (a); significant correlation between flood and post-flood season, panel (b); see Sect. 4.3): relative differences (\%) between the 100-yr peak flows estimated independently through a maximum likelihood estimator, or retrieved from jointly estimated seasonal CDFs by adopting uniform weights (proposed technique); the two boxplots for the ANNUAL case present the comparison between the 100-yr flood estimated at annual timescale through a maximum likelihood estimator and the corresponding estimates retrieved from CDFs obtained as the product of jointly estimated seasonal CDFs (proposed approach - blue boxplot), or the product of independently estimated seasonal CDFs (light blue boxplot).

for the independent subdivisions (panel a), which is a further conformation of the validity of the proposed approach.

\section{Discussion and concluding remarks}

We propose an estimation procedure for the joint fitting of seasonal and annual flood frequency distributions that ensures their consistency, i.e. the fact that the probability of one peak value of being exceeded in the entire year is higher than or equal to the probability of the same value of being exceeded in one season. The main assumption of the method is the hypothesis of statistical independence among seasons. Thus, strictly speaking, the method should be applied only if the hypothesis of independence cannot be rejected (for an assigned significance level).

A relevant feature of the approach is that the number of seasons and their calendar limits can be defined with great flexibility. However, this characteristic is limited by the main assumption of the approach, as the statistical dependence among seasons generally increases with the number of seasons. Also, differently from previous studies, our method enables the user to attribute weights to the estimation of seasonal and/or annual flood frequency distributions.
The approach was applied to the Blue Nile River at SudanEthiopia Border for the estimation of seasonal and annual flood quantiles. We performed a comprehensive analysis to assess the sensitivity of the proposed method to the seasonal weighting scheme that is used in the optimisation procedure, and to the selected number and duration of seasons, as well as the degree of their mutual dependence. The results of the analysis show for the considered case study that the use of weights enables the user to obtain a seasonal or annual flood frequency distribution very close to the corresponding maximum likelihood estimate. Also, since the method accounts for the dependence among seasonal and annual maxima (and not among the seasons themselves), the annual distribution, as well as the dominant seasons, are sensitive to the value of the weights, while the not-dominant seasons (the dry season in our case) show little or no sensitivity. Furthermore, the analysis showed that, regardless of the degree of statistical dependence between seasons, the product of the jointlyestimated seasonal cumulative distribution functions (CDFs) is always a better approximation to the local CDF of annual maxima than the product of seasonal CDFs estimated independently of each other through a maximum likelihood estimator.

Further research work is currently under development to test the approach in different climatic and geographic regions and to relax the main assumption of independence among seasons, therefore ensuring full flexibility of the approach for practical applications. 
Acknowledgements. This work has been partially supported by the Italian government through the grant "Uncertainty estimation for precipitation and river discharge data. Effects on water resources planning and flood risk management".

The insightful and thoughtful comments of Hessel Winsemius and an anonymous referee are thankfully acknowledged. We are certainly indebted to the editor Dominic Mazvimavi, for his additional and careful rereading of our manuscript, which enabled us to include some further corrections.

Edited by: D. Mazvimavi

\section{References}

Allamano, P., Laio, F., and Claps, P.: Effects of disregarding seasonality on the distribution of hydrological extremes, Hydrol. Earth Syst. Sci., 15, 3207-3215, doi:10.5194/hess-15-32072011, 2011.

Bayliss, A. C. and Jones, R. C.: Peaks-over-threshold flood database: summary statistics and seasonality, Crowmarsh Gifford, Rep. 121, Institute of Hydrology, Wallingford, 61 pp., 1993.

Bowers, M. C., Tung, W. W., and Gao, J. B.: On the distributions of seasonal river flows: lognormal or power law?, Water Resour. Res., 48, W05536, doi:10.1029/2011WR011308, 2012.

Buishand, T. A. and Demarè, G. R.: Estimation of the annual maximum distribution from samples of maxima in separate seasons, Stoch. Hydrol. Hydraul., 4, 89-103, doi:10.1007/BF01543284, 1990.

Burn, D. H.: Catchment similarity for regional flood frequency analysis using seasonality measures, J. Hydrol., 202, 212-230, doi:10.1016/S0022-1694(97)00068-1, 1997.

Castellarin, A., Burn, D. H., and Brath, A.: Assessing the effectiveness of hydrological similarity measures for flood frequency analysis, J. Hydrol., 241, 270-285, doi:10.1016/S00221694(00)00383-8, 2001.

Castellarin, A., Vogel, R. M., and Brath, A.: A stochastic index flow model of flow duration curves, Water Resour. Res., 40, W03104, doi:10.1029/2003WR002524, 2004.

Chen, L., Guo, S., Yan, B., Liu, P., and Fang, B.: A new seasonal design flood method based on bivariate joint distribution of flood magnitude and date of occurrence, Hydrolog. Sci. J., 55, 12641280, doi:10.1080/02626667.2010.520564, 2010.

Creager, W. P., Kinnison, H. B., Shifrin, H., Snyder, F. F., Williams, G. R., Gumbel, E. J., and Matthes, G. H.: Review of flood frequency methods: final report of the subcommittee of the joint division committee on floods, Trans. ASCE, 116, 12201230, 1951.

Cunderlik, J. and Burn, D. H.: Analysis of the linkage between rain and flood regime and its application to regional flood frequency estimation, J. Hydrol., 261, 115-131, 2002.

Durrans, S., Eiffe, M., Thomas, W., and Goranflo, H.: Joint Seasonal/Annual Flood Frequency Analysis, J. Hydol. Eng., 8, 181189, doi:10.1061/(ASCE)1084-0699(2003)8:4(181), 2003.
Elshamy, M. E., Seierstad, I. A., and Sorteberg, A.: Impacts of climate change on Blue Nile flows using bias-corrected GCM scenarios, Hydrol. Earth Syst. Sci., 13, 551-565, doi:10.5194/hess13-551-2009, 2009.

Fang, B., Guo, S., Wang, S., Liu, P., and Xiao, Y.: Non-identical models for seasonal flood frequency analysis, Hydrolog. Sci. J., 52, 974-991, doi:10.1623/hysj.52.5.974, 2007.

Kochanek, K., Strupczewski, W. G., and Bogdanowicz, E.: On seasonal approach to flood frequency modelling, Part II: Flood frequency analysis of Polish rivers, Hydrol. Process., 26, 717-730, doi:10.1002/hyp.8178, 2012.

Madsen, H., Rasmussen, P. F., and Rosbjerg, D.: Comparison of annual maximum series and partial duration series methods for modeling extreme hydrologic events: 1 . At-site modeling, Water Resour. Res., 33, 747-757, doi:10.1029/96WR03848, 1997a.

Madsen, H., Pearson, C. P., and Rosbjerg, D.: Comparison of annual maximum series and partial duration series methods for modeling extreme hydrologic events: 2. Regional modeling, Water Resour. Res., 33, 759-769, doi:10.1029/96WR03849, $1997 \mathrm{~b}$.

Mardia, K. V.: Statistics of Directional Data, Academic, San Diego, Calif., 1972.

McCuen, R. H. and Beighley, R. E.: Seasonal flow frequency analysis, J. Hydrol., 279, 43-56, doi:10.1016/S0022-1694(03)001549, 2003.

Mebane Jr., W. R. and Sekhon, J. S.: Rgenoud: genetic optimization using derivatives: the rgenoud package for R, J. Stat. Softw., 42, 1-26, 2011.

Rientjes, T. H. M., Haile, A. T., Kebede, E., Mannaerts, C. M. M., Habib, E., and Steenhuis, T. S.: Changes in land cover, rainfall and stream flow in Upper Gilgel Abbay catchment, Blue Nile basin - Ethiopia, Hydrol. Earth Syst. Sci., 15, 1979-1989, doi:10.5194/hess-15-1979-2011, 2011.

Seleshi, Y. and Camberlin, P.: Recent changes in dry spell and extreme rainfall events in Ethiopia, Theor. Appl. Climatol., 83, 181-191, doi:10.1007/s00704-005-0134-3, 2006.

Singh, V. P., Wang, S. X., and Zhang, L.: Frequency analysis of nonidentically distributed hydrologic flood data, J. Hydrol., 307, 175-195, doi:10.1016/j.jhydrol.2004.10.029, 2005.

Stedinger, J. R., Vogel, R. M., and Foufoula-Georgiou, E.: Frequency analysis of extreme events, Chapter 18, Handbook of Hydrology, edited by: Maidment, D. R., McGraw-Hill, New York, 1993.

Strupczewski, W. G., Kochanek, K., Bogdanowicz, E., and Markiewicz, I.: On seasonal approach to flood frequency modelling, Part I: flood frequency analysis of Polish rivers, Hydrol. Process., 26, 705-716, doi:10.1002/hyp.8179, 2012.

Vogel, R. M.: The probability plot correlation coefficient test for the normal, log-normal and Gumbel distributional hypothesis, Water Resour. Res., 22, 587-590, doi:10.1029/WR022i004p00587, 1986.

Waylen, P. and Woo, M. K.: Prediction of annual floods generated by mixed processes, Water Resour. Res., 18, 1283-1286, doi:10.1029/WR018i004p01283, 1982. 